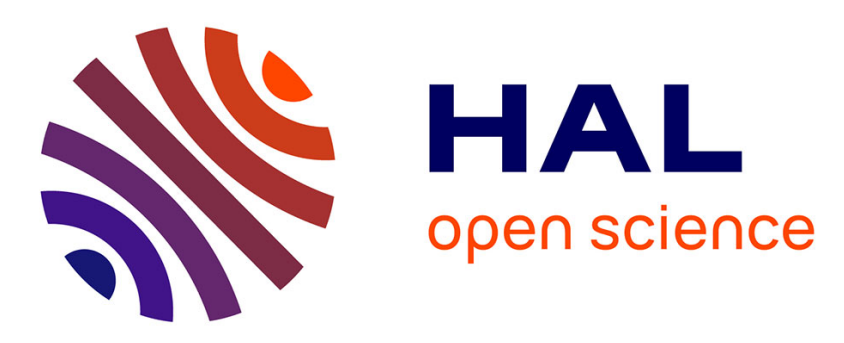

\title{
Three-dimensional finite-difference time-domain algorithm for oblique incidence with adaptation of perfectly matched layers and nonuniform meshing: Application to the study of a radar dome
}

\author{
A. Belkhir, F.I. Baida
}

\section{To cite this version:}

A. Belkhir, F.I. Baida. Three-dimensional finite-difference time-domain algorithm for oblique incidence with adaptation of perfectly matched layers and nonuniform meshing: Application to the study of a radar dome. Physical Review E: Statistical, Nonlinear, and Soft Matter Physics, 2008, 77 (5), pp.056701. 10.1103/PhysRevE.77.056701 . hal-00282823

\section{HAL Id: hal-00282823 \\ https://hal.science/hal-00282823}

Submitted on 11 May 2021

HAL is a multi-disciplinary open access archive for the deposit and dissemination of scientific research documents, whether they are published or not. The documents may come from teaching and research institutions in France or abroad, or from public or private research centers.
L'archive ouverte pluridisciplinaire HAL, est destinée au dépôt et à la diffusion de documents scientifiques de niveau recherche, publiés ou non, émanant des établissements d'enseignement et de recherche français ou étrangers, des laboratoires publics ou privés. 


\title{
Three-dimensional finite-difference time-domain algorithm for oblique incidence with adaptation of perfectly matched layers and nonuniform meshing: Application to the study of a radar dome
}

\author{
A. Belkhir* and F. I. Baida ${ }^{\dagger}$ \\ Département d'Optique P.M. Duffieux, Institut FEMTO-ST UMR 6174 CNRS, Université de Franche-Comté, \\ 25030 Besançon Cedex, France
}

(Received 22 October 2007; published 2 May 2008)

\begin{abstract}
The three-dimensional finite-difference time-domain (3D-FDTD) method is developed and implemented in the case of oblique incidence in order to study biperiodic structures that are finished according to the third direction. The perfectly matched layer (PML) is adapted to the developed algorithm. The electromagnetic fields of Maxwell's equations in the main grid and in the PML media are transferred from the $E-H$ domain to the mapped $P-Q$ domain. The modified Maxwell's equations are implemented by the split-field method (SFM). Several tests are made and presented in order to verify and demonstrate the accuracy of our codes. The obtained results are in good agreement with published ones obtained by other methods. The originality of this paper comes, first from the fact that it brings a complete development of the used algorithm, and second, from the study of the spectral response of a radar dome based on annular aperture arrays perforated into a perfect conductor plate.
\end{abstract}

DOI: 10.1103/PhysRevE.77.056701

PACS number(s): 02.70.Bf, 42.25.Bs, 02.70.-c, 02.60.Cb

\section{INTRODUCTION}

The dielectric and metallic periodic structures have recently attracted wide attention for various applications in nanotechnology such as electromagnetic band-gap structures $[1,2]$, enhanced transmission [3,4], frequency selective surfaces $[5,6], \ldots$. The study and the design of these structures require numerical models before making expensive and difficult experiments. These methods are numerous: a lot of them are frequential methods such as the Fourier modal method (FMM), the finite element method (FEM), and the method of moment (MOM). The wide band analysis by these methods requires one to carry out one study by frequency. In addition, some of these methods such as FMM and MOM are limited to the study of structures having simple geometries. Methods that work in the time domain are then more attractive for the spectral analysis. Let us quote, for example, the finite-difference time domain (FDTD), used in this paper, and the FEM method working in the time domain. Using these time-domain methods, a wide band characterization can be obtained from one temporal calculation via a simple Fourier transform.

The FDTD method, introduced by Yee, is a powerful and general tool for the resolution of differential equations system [7]. It is easy to be numerically implemented [8]. The implementation is based on the discretization of the differential equations by means of centered finite differences. In principle, it can take into account any geometry. For the normal incidence case, the FDTD method is widely used to study the periodic structures. In this case, the periodic boundary conditions (PBCs), i.e., the Floquet-Block conditions, can be applied easily without any modification of the

\footnotetext{
*Permanent address: Laboratoire de Physique et Chimie Quantique, Université Mouloud Mammeri, Tizi-Ouzou, Algeria.

${ }^{\dagger}$ Corresponding author; fbaida@univ-fcomte.fr
}

traditional Yee's schema [9-11]. For the oblique incidence, the application of the PBCs explicitly reveals the frequency of the electromagnetic field. This is in contradiction with the temporal character of the FDTD algorithm. To adapt the FDTD in order to treat such a problem (the oblique incidence), Veysoglu et al. introduced the field transformation method (FTM) [12]. Thus, the field components in Maxwell's equations are transferred from the $E-H$ domain to the mapped $P-Q$ domain. The direct implementation of the modified Maxwell's equations without modifying the Yee schema remains impossible. Several techniques of implementation were proposed (see Chapter 13 of Ref. [8]), for example, the split-field method (SFM) [13] used in this work, and the exponential time differencing (ETD) proposed recently by [14]. Since Veysegol, several researchers worked on the FDTD in the oblique incidence case by using the SFM technique. Wu et al. studied the photonic crystal filters by the FDTD in oblique incidence. Oh and Escuti implemented the two-dimensional (2D) FDTD for the oblique analysis of anisotropic periodic structures [15].

The work presented in this paper is a complete 3D-FDTD implementation in the oblique incidence using the SFM for the analysis of the biperiodic structures that are finished according to the third direction. The developed algorithm is especially devoted to the study of the enhanced transmission through nanoapertures engraved into metallic layer or to the study of finite 3D photonic crystals, chiefly, the determination of the dispersion relation for such structure.

The electromagnetic field components in Maxwell's equations are transferred from the $E-H$ domain to the mapped $P-Q$ domain. The modified Maxwell's equations are discretized with centered differences and then implemented by the SFM. The open medium into the third direction is delimited by perfectly matched layers (PML) that are also adapted for the oblique case. The equations in the PML media are also modified, expressed in the mapped $(P-Q)$ domain and implemented using the SFM method. 


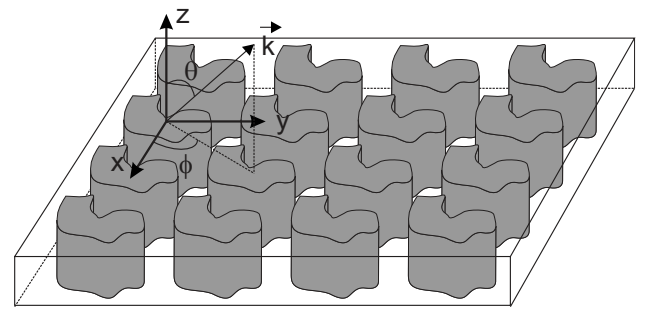

FIG. 1. Sketch of the biperiodic structure that is illuminated by a plane wave propagating along the $\vec{k}$ vector defined by its Euler angles $\theta$ and $\phi$.

The validation of our code is provided via a set of tests that consists, first, in an analytical verification of the reflection and transmission coefficients (Fresnel coefficients). Second, a $2 \mathrm{D}$ diffraction problem is studied in the case of a perfect metal according to the incidence angle and to the polarization. Finally, a 3D problem is also examined in the case of a dielectric structure versus the polarization, the angle of incidence, and the azimuthal angle. For both these two last cases (2D and 3D), comparisons with already published results in TE and TM polarizations are done $[16,17]$. Before the conclusion, we apply our codes to extend the study on the enhanced transmission through annular aperture arrays presented by Van Labeke et al. [6] to the case of a perfect conductor in order to design a metallic radar radome (a hull or dome used to protect or to hid a radar or an antenna).

We emphasize here the fact that, in spite of the existence of some papers where the basis of the method are already published, the originality of our paper comes from the fact that two treated examples are veritable $3 \mathrm{D}$ structures that need full 3D calculations. In most of the published studies, the handled objects are two dimensional such as lamellar gratings or infinitely thin periodic arrays [18]. In Ref. [14], 3D calculations in oblique incidence are carried out for applications in the microwave domain. In that paper, the ETD technic is employed but the modified PML equations are not presented in addition to the fact that this technic cannot be extended to the case of dispersive materials. In Ref. [15], the SFM technic is developed for anisotropic media but no 3D simulations are presented.

The final part of this paper is devoted to an original study of a 3D periodic structure where both the azimuthal and the incidence angles vary. This study leads to results allowing the design of a radome based on annular aperture arrays pierced into metallic screens.

\section{FDTD FORMULATION IN OBLIQUE INCIDENCE}

The structure under study is a biperiodic array along the two directions $x$ and $y$ (see Fig. 1). It is limited along the third direction $(z)$. Let us consider a plane wave propagation along the $\vec{k}$ vector as described in Fig. 1 .

The direction of propagation, i.e., the direction of the $\vec{k}$ vector, is defined in the (xyz) coordinates system by the Euler angles $\phi$ and $\theta$. The electric and magnetic fields of the incident electromagnetic field can be written as

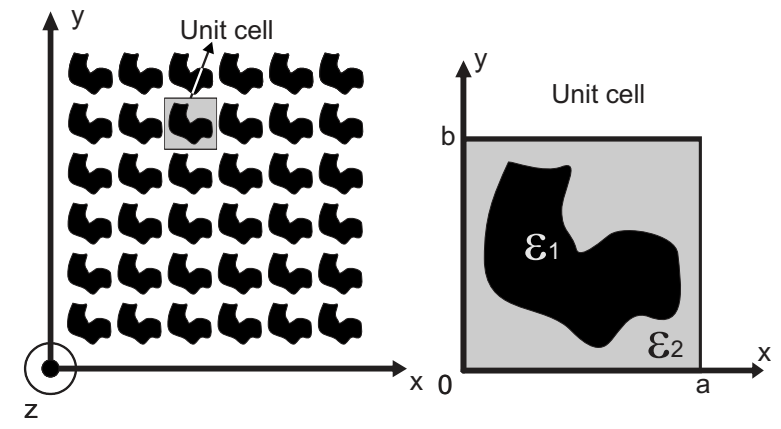

FIG. 2. Schematic of a biperiodic structure and the unit cell used in the FDTD calculations.

$$
\begin{aligned}
\vec{E} & =\vec{E}_{0} e^{j\left(k_{x} x+k_{y} y+k_{z} z\right)}, \\
\vec{H} & =\vec{H}_{0} e^{j\left(k_{x} x+k_{y} y+k_{z} z\right)},
\end{aligned}
$$

where $k_{x}=\frac{\omega}{v_{i}} \sin \theta \cos \phi, k_{y}=\frac{\omega}{v_{i}} \sin \theta \sin \phi, k_{z}=\frac{\omega}{v_{i}} \cos (\theta)$, and $v_{i}$ is the light speed in the incident medium.

For the study of periodic structures such as photonic crystals (see Fig. 2), only one elementary cell can be considered to describe the behavior of the infinite structure. This can be obtained via the periodic boundary conditions (PBCs) of Floquet-Bloch that are imposed to the electric and magnetic components.

These PBCs are then written as following:

$$
\begin{gathered}
\vec{E}(x+a, y, z, t)=\vec{E}(x, y, z, t) e^{j k_{x} a}, \\
\vec{E}(x, y+b, z, t)=\vec{E}(x, y, z, t) e^{j k_{y} b}, \\
\vec{H}(x, y, z, t)=\vec{H}(x+a, y, z, t) e^{-j k_{x} a}, \\
\vec{H}(x, y, z, t)=\vec{H}(x, y+b, z, t) e^{-j k_{y} b},
\end{gathered}
$$

where $a$ and $b$ are the periodicity in the $x$ and $y$ directions, respectively. These latest equations explicitly depend on the value of $\omega$ through both $k_{x}$ and $k_{y}$. Accordingly, they cannot be directly used in the FDTD algorithm because this later operates in the time domain and not in the frequency one. To bypass this $\omega$ dependence in the phase terms, a new set of field variables is introduced:

$$
\begin{aligned}
& \vec{P}=\vec{E} e^{-j\left(k_{x} x+k_{y} y\right)}, \\
& \vec{Q}=\vec{H} e^{-j\left(k_{x} x+k_{y} y\right)} .
\end{aligned}
$$

The periodic boundary conditions for the transformed field variables can now be expressed as

$$
\begin{aligned}
& \vec{P}(x+a, y, z, t)=\vec{P}(x, y, z, t), \\
& \vec{P}(x, y+b, z, t)=\vec{P}(x, y, z, t), \\
& \vec{Q}(x, y, z, t)=\vec{Q}(x+a, y, z, t),
\end{aligned}
$$




$$
\vec{Q}(x, y, z, t)=\vec{Q}(x, y+b, z, t) .
$$

The modified Maxwell's equations in the mapped $P-Q$ domain are obtained by injecting Eqs. (3) in the classical Maxwell's equations explained in the $E-H$ domain.

In order to simplify the numerical FDTD implementation, most FDTD codes (especially the commercial codes) are built in a general way that assumes a PML media for all the computational domain (maingrid+PML). This avoids the special processing of the flat interfaces between the main grid and the PML. Thereby, the electric conductivity $\sigma$ and magnetic loss $\sigma^{*}$ are fixed to zero in the main grid while their values increase in the PML regions. This technique is highly memory and time consuming because each field component is split in two for the PML medium. For this last reason, we have developed our code to treat separately the two parts with additional equations that connect the two media. The implementation of the FTM+SFM for the main grid is already presented in several works (see Ref. [8] and references therein). Consequently, only the implementation of the modified PML equations will be presented in the following; the main grid equations can be easily obtained by replacing $\sigma$ and $\sigma^{*}$ by zero.

It is clear that, because of the $x$ and $y$ periodicity, the PML has to be implemented only in the $z$ direction. As mentioned above, each field component is split in two parts except for the $z$ component. Thus, in the PML $\vec{E}$ (or $\vec{H}$ ) has five components. Let $E_{x y}, E_{x z}, E_{y x}, E_{y z}$, and $E_{z}$ be these components (idem for $\vec{H}$ ). These field components are then transferred from the $E-H$ domain to the mapped $P-Q$ domain through Eqs. (3).

By replacing the $E$ and $H$ components of Eqs. (3) in the classical Maxwell's equations, one obtains the modified equations expressed in the mapped $(P-Q)$ domain,

$$
\begin{gathered}
\epsilon \frac{\partial P_{x y}}{\partial t}=\frac{\partial Q_{z}}{\partial y}+j \frac{\omega}{v_{i}} \sin \theta \sin \phi Q_{z}, \\
\left(\epsilon \frac{\partial}{\partial t}+\sigma_{z}\right) P_{x z}=-\frac{\partial}{\partial z}\left(Q_{y x}+Q_{y z}\right), \\
\left(\epsilon \frac{\partial}{\partial t}+\sigma_{z}\right) P_{y z}=\frac{\partial}{\partial z}\left(Q_{x y}+Q_{x z}\right), \\
\epsilon \frac{\partial P_{y x}}{\partial t}=-\frac{\partial Q_{z}}{\partial x}-j \frac{\omega}{v_{i}} \sin \theta \cos \phi Q_{z}, \\
\epsilon \frac{\partial P_{z}}{\partial t}=\frac{\partial}{\partial x}\left(Q_{y x}+Q_{y z}\right)-\frac{\partial}{\partial y}\left(Q_{x y}+Q_{x z}\right) \\
+j \frac{\omega}{v_{i}} \sin \theta \sin \phi\left(Q_{y x}+Q_{y z}\right) \\
-j \frac{\omega}{v_{i}} \sin \theta \cos \phi\left(Q_{x y}+Q_{x z}\right), \\
\mu \frac{\partial Q_{x y}}{\partial t}=-\frac{\partial P_{z}}{\partial y}-j \frac{\omega}{v_{i}} \sin \theta \sin \phi P_{z},
\end{gathered}
$$

$$
\begin{gathered}
\left(\mu \frac{\partial}{\partial t}+\sigma_{z}^{*}\right) Q_{x z}=\frac{\partial}{\partial z}\left(P_{y x}+P_{y z}\right), \\
\left(\mu \frac{\partial}{\partial t}+\sigma_{z}^{*}\right) Q_{y z}=-\frac{\partial}{\partial z}\left(P_{x y}+P_{x z}\right), \\
\mu \frac{\partial Q_{y x}}{\partial t}=\frac{\partial P_{z}}{\partial x}+j \frac{\omega}{v_{i}} \sin \theta \cos \phi P_{z}, \\
\mu \frac{\partial Q_{z}}{\partial t}=\frac{\partial}{\partial y}\left(P_{x y}+P_{x z}\right)-\frac{\partial}{\partial x}\left(P_{y x}+P_{y z}\right) \\
\quad+j \frac{\omega}{v_{i}} \sin \theta \sin \phi\left(P_{x y}+P_{x z}\right) \\
-j \frac{\omega}{v_{i}} \sin \theta \cos \phi\left(P_{y x}+P_{y z}\right) .
\end{gathered}
$$

One should notice that in the case of normal incidence $(\theta=0)$, the previously modified equations turn into the traditional PML equations of Bérenger explained in the $E-H$ domain. However, in oblique case, the additional terms (where $\omega$ arises explicitly) are equivalent to additional timederivative terms (ATDTs) that appear on the right-hand side of Eqs. (5). These ATDT terms need a special treatment to be implemented. In fact, they can be bypassed by splitting the field components as

$$
\begin{gathered}
P_{x y}=P_{x y a}+\frac{\sin \theta \sin \phi}{v_{i} \epsilon} Q_{z} \\
P_{x z}=P_{x z a}, \\
P_{y z}=P_{y z a}, \\
P_{y x}=P_{y x a}-\frac{\sin \theta \cos \phi}{v_{i} \epsilon} Q_{z}, \\
P_{z}=P_{z a}+\frac{\sin \theta \cos \phi}{v_{i} \epsilon}\left(Q_{y x}+Q_{y z}\right)-\frac{\sin \theta \sin \phi}{v_{i} \epsilon}\left(Q_{x y}+Q_{x z}\right), \\
Q_{x y}=Q_{x y a}-\frac{\sin \theta \sin \phi}{v_{i} \mu} P_{z} \\
Q_{x z}=Q_{x z a}, \\
Q_{y z}=Q_{y z a}, \\
Q_{y x}=Q_{y x a}+\frac{\sin \theta \cos \phi}{v_{i} \mu} P_{z}
\end{gathered}
$$

$$
Q_{z}=Q_{z a}+\frac{\sin \theta \sin \phi}{v_{i} \mu}\left(P_{x y}+P_{x z}\right)-\frac{\sin \theta \cos \phi}{v_{i} \mu}\left(P_{y x}+P_{y z}\right) .
$$


One can see from Eqs. $(6 \mathrm{~b}),(6 \mathrm{c}),(6 \mathrm{~g})$, and (6h) that it is not necessary to split the components $P_{x z}, P_{y z}, Q_{x z}$, and $Q_{y z}$.

Substituting Eqs. (6) into the left-hand side of Eqs. (5) results in equations that allow the calculations of the $a$ fields through the total fields,

$$
\begin{gathered}
\epsilon \frac{\partial P_{x y a}}{\partial t}=\frac{\partial Q_{z}}{\partial y}, \\
\left(\epsilon \frac{\partial}{\partial t}+\sigma_{z}\right) P_{x z a}=-\frac{\partial}{\partial z}\left(Q_{y x}+Q_{y z}\right), \\
\left(\epsilon \frac{\partial}{\partial t}+\sigma_{z}\right) P_{y z a}=\frac{\partial}{\partial z}\left(Q_{x y}+Q_{x z}\right), \\
\epsilon \frac{\partial P_{y x a}}{\partial t}=-\frac{\partial Q_{z}}{\partial x}, \\
\epsilon P_{z a}=\frac{\partial}{\partial x}\left(Q_{y x}+Q_{y z}\right)-\frac{\partial}{\partial y}\left(Q_{x y}+Q_{x z}\right), \\
\mu \frac{\partial Q_{x y a}}{\partial t}=-\frac{\partial P_{z}}{\partial y}, \\
\mu \frac{\partial Q_{y x a}}{\partial t}=\frac{\partial P_{z}}{\partial x}, \\
\left(\mu \frac{\partial}{\partial t}+\sigma_{z}^{*}\right) Q_{x z a}=\frac{\partial}{\partial z}\left(Q_{y x}+Q_{y z}\right), \\
\left(\mu \frac{\partial}{\partial t}+\sigma_{z y}^{*}+P_{x z}\right)-\frac{\partial}{\partial x}\left(P_{y x}+P_{y z}\right) . \\
\frac{\partial}{\partial z}\left(P_{x y}+P_{x z}\right),
\end{gathered}
$$

The numerical calculation of the components of $\vec{P}_{a}$ and $\vec{Q}_{a}$ is done through the time and space discretization of the last system after replacing the time and space derivatives by centered finite differences. For example, the $P_{x y a}$ component is given by

$$
\begin{aligned}
P_{x y a}^{n}(i, j, k)= & P_{x y a}^{n-1}(i, j, k) \\
& +\frac{\partial t}{\epsilon}\left(\frac{Q_{z}^{n-1 / 2}(i, j, k)-Q_{z}^{n-1 / 2}(i, j-1, k)}{\Delta y}\right) .
\end{aligned}
$$

To obtain the upgraded values of the total field components $(\vec{P}$ and $\vec{Q})$, we first substitute Eqs. (6a)-(6d) in Eq. (6j). Second, we also substitute (6f)-(6i) in Eq. (6e). After some algebra, we obtain

$$
\begin{aligned}
& P_{z}^{n}=\frac{1}{\left(1-\frac{\sin ^{2} \theta \cos ^{2} \phi}{\epsilon \mu v_{i}^{2}}-\frac{\sin ^{2} \theta \sin ^{2} \phi}{\epsilon \mu v_{i}^{2}}\right)} \\
& \times\left(P_{z a}^{n}+\frac{\sin \theta \cos \phi}{\epsilon v_{i}}\left(Q_{y x a}^{n}+Q_{y z a}^{n}\right)\right. \\
& \left.-\frac{\sin \theta \sin \phi}{\epsilon v_{i}}\left(Q_{x y a}^{n}+Q_{x z a}^{n}\right)\right), \\
& Q_{z}^{n}=\frac{1}{\left(1-\frac{\sin ^{2} \theta \cos ^{2} \phi}{\epsilon \mu v_{i}^{2}}-\frac{\sin ^{2} \theta \sin ^{2} \phi}{\epsilon \mu v_{i}^{2}}\right)} \\
& \times\left(Q_{z a}^{n}+\frac{\sin \theta \sin \phi}{\mu v_{i}}\left(P_{x y a}^{n}+P_{x z a}^{n}\right)\right. \\
& \left.-\frac{\sin \theta \cos \phi}{\mu v_{i}}\left(P_{y x a}^{n}+P_{y z a}^{n}\right)\right), \\
& P_{x y}^{n}=P_{x y a}^{n}+\frac{\sin \theta \sin \phi}{\epsilon v_{i}} Q_{z}^{n}, \\
& P_{x z}^{n}=P_{x z a}^{n}, \\
& P_{y z}^{n}=P_{y z a}^{n}, \\
& P_{y x}^{n}=P_{y x a}^{n}-\frac{\sin \theta \cos \phi}{\epsilon v_{i}} Q_{z}^{n}, \\
& Q_{x y}^{n}=Q_{x y a}^{n}-\frac{\sin \theta \sin \phi}{\mu v_{i}} P_{z}^{n}, \\
& Q_{x z}^{n}=Q_{x z a}^{n}, \\
& Q_{y z}^{n}=Q_{y z a}^{n}, \\
& Q_{y x}^{n}=Q_{y x a}^{n}+\frac{\sin \theta \cos \phi}{\mu v_{i}} P_{z}^{n} .
\end{aligned}
$$

A two time-step updating algorithm is used to update both Eqs. (9) and the resulting system after discretization of Eqs. (7).

On the contrary of a conventional Yee's schema, all the field components are calculated at all time steps. Thus the components of $\vec{P}, \vec{Q}, \vec{P}_{a}$, and $\vec{Q}_{a}$, have to be determined at $t=n \Delta t$, and $t=(n+1 / 2) \Delta t$ where $\Delta t$ is the time step.

Finally, we remind the reader of the stability criteria that induce a maximum value for the temporal step given by [19] 


$$
\Delta t_{\max }=\frac{\min (\Delta x, \Delta y, \Delta z)\left(v_{i}^{2} \mu \epsilon-\sin ^{2} \theta\right)}{v_{i}\left[|\sin \theta \cos \phi|+|\sin \theta \sin \phi|+\sqrt{3 v_{i}^{2} \mu \epsilon-2 \sin ^{2} \theta(1-|\sin \phi \cos \phi|)}\right.} .
$$

The next section of our paper is devoted to the validation of our codes.

\section{DEMONSTRATION OF OUR CODE ACCURACY}

In order to validate the implemented codes, we will present in this paragraph three different tests on the calculation of light diffraction by $0 \mathrm{D}, 1 \mathrm{D}$, and $2 \mathrm{D}$ periodic struc-
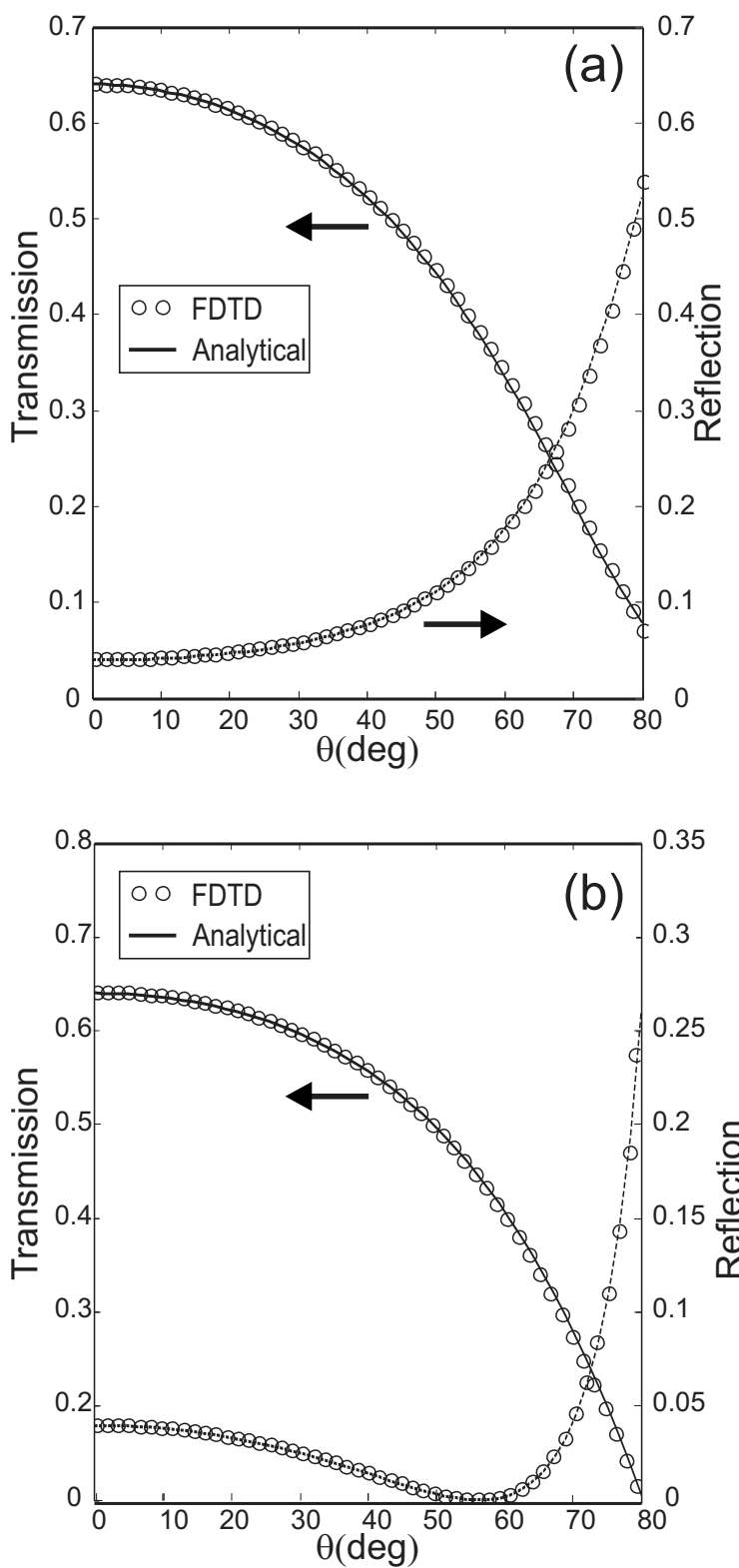

tures. The $0 \mathrm{D}$ object corresponds to a flat interface separating two different media, the 1D case is associated with the diffraction problem by a $1 \mathrm{D}$ periodic structure (periodic in $x$, infinite in $y$, and finite in $z$ ), while the 2D case corresponds to a biperiodic structure in the $x$ and the $y$ directions and finite along the $z$ one. Note here that the PML [20] implementation uses absorption coefficients that vary spatially according to a polynomial law given by
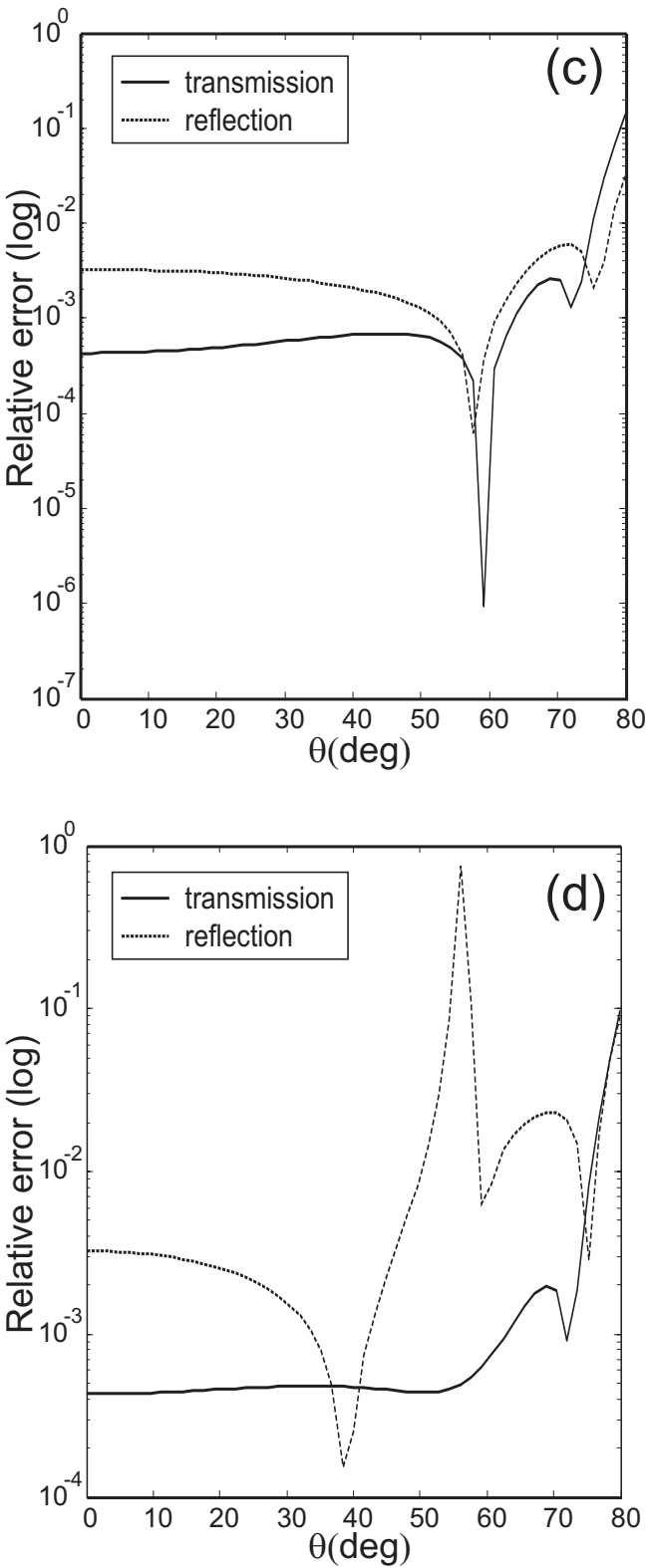

FIG. 3. Transmission and reflection coefficients versus the angle of incidence for a flat vacuum-glass interface. (a),(c) TE polarization; (b),(d) TM polarization. Comparisons between analytical and FDTD coefficients are presented in (a) and (b), while the relative errors are depicted in (c) and (d), respectively. 

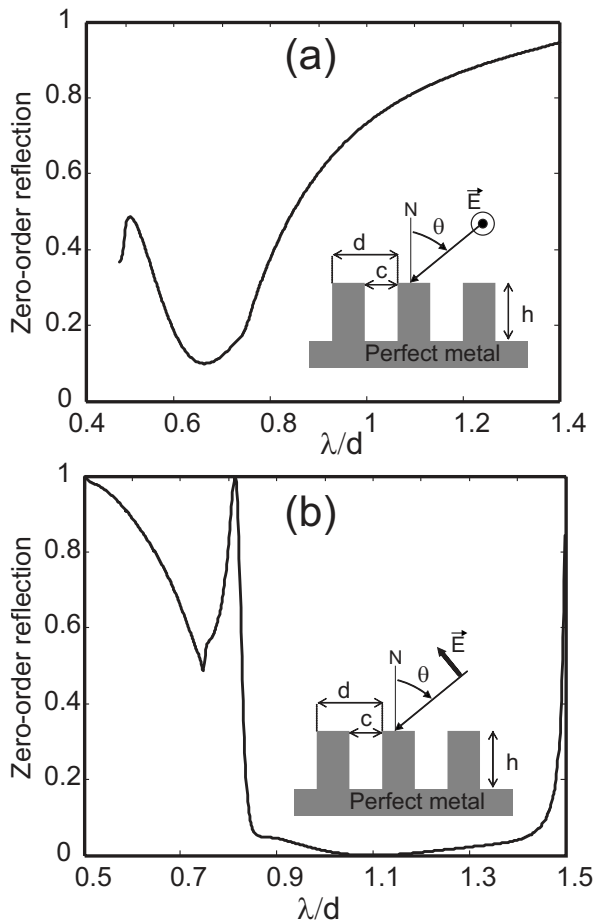

FIG. 4. Zero-order reflection coefficients of a lamellar perfectly conducting grating for the both TE in (a) and TM in (b) polarizations for $\theta=30^{\circ}$.

$$
\sigma(z)=\sigma_{m}\left[\frac{z-z_{P M L}}{N \Delta z}\right]^{m},
$$

where $m$ is the polynomial order fixed to 2 in this work, $z_{P M L}$ is the $z$ position of the main grid-PML interface, $N$ is the number of the PML layers set to 10 , and $\Delta z$ is the spatial step according to the $z$ direction. $\sigma_{m}$ is the maximum value of $\sigma(z)$ that occurs at the external edge of the PML area. Throughout all the presented simulations, the value of $\sigma_{m}$ is fixed to $6.42 \times 10^{-3} \mathrm{~S} / \mathrm{m}$.

\section{A. The $0 \mathrm{D}$ test}

This first test consists to numerically calculate the transmission or the reflection coefficient of a linearly polarized plane wave illuminating a flat interface. The two considered media are vacuum $(\varepsilon=1)$ and glass $(\varepsilon=2.25)$. The two polarizations [TE and transverse magnetic (TM)] are studied. Figure 3 shows the FDTD results in comparison with the well-known analytical results of the transmission and the reflection versus the angle of incidence. The relative error calculated as $\frac{\left|T_{F D T D}-T\right|}{T}$, where $T$ is the analytical value of the transmission, is also presented in the same figure and shows a maximum of around $1 \times 10^{-3}$ for $\theta<60^{\circ}$. This last value is small enough to ensure a good accuracy of our code; its value depends on the PML parameters that can be optimized by increasing the PML layer number and/or by decreasing the spatial step.

\section{B. The $1 \mathrm{D}$ test}

In order to compare our FDTD results with already published ones, we consider a lamellar perfectly conducting grating that was studied in Ref. [16] on page 162. The zeroorder reflected efficiencies calculated by our code are shown in Fig. 4 for TE and TM polarizations. In both cases, the angle of incidence was fixed to $30^{\circ}$ and the other geometrical parameters are given by the insets appearing in each figure part.

As it can be noted, a very good agreement is obtained between our results and the ones of Fig 6.2a and 6.2b of Ref. [16]. In that reference, a differential method was used to perform the spectral responses of the structure and comparison with experimental results were also done.

\section{The 2D test}

This section is devoted to the study of a structure that is biperiodic along the $x$ and $y$ directions and finite in the $z$ direction. According to us, this being the decisive test because it consists of a general study where different parameters for the illumination are considered. In fact, and in addition to the polarization and the angle of incidence, the influence of the azimuthal angle is also investigated. For this purpose, we consider the two-dimensional dielectric grating studied in Refs. [17,21] and we compare our results with published ones that were obtained by a vectorial modal method (VMM), also named modal analysis by the authors.

We have fixed our geometrical parameters according to the ones of Ref. [17]. That leads one to set the pattern of the unit cell of Fig. 2 to a parallelepiped having $h=2 \mathrm{~mm}$ height and a square section with sides of $10 \times 10 \mathrm{~mm}^{2}$ parallel to the $x$ and $y$ axes. The periods along the $x$ and $y$ directions are fixed to $a=b=20 \mathrm{~mm}$. The dielectric permittivity is equal to $\varepsilon_{1}=4$ for the parallelepiped and it is set to $\varepsilon_{2}=10$ for the background. The structure is supposed to be surrounded by vacuum from both the top and the bottom sides.

The first study consists in the calculations of the frequential response of the specular reflected energy for both TE and TM polarizations with $\phi=0^{\circ}$. This means that the incidence plane, which is defined by the incident wave vector and the normal to the surface, is parallel to the $x$ axis (see Fig. 1).

The calculated specular reflectivities for $\theta=0^{\circ}, \theta=15^{\circ}$, and $\theta=30^{\circ}$ both in TE and TM are presented in Fig. 5. Comparisons with results obtained by the VMM method are done and show small discrepancies, especially in the TM case. In addition, for $\theta=0^{\circ}$ and TM polarization [Fig. 5(a)], three other theoretical results are depicted and compared with our FDTD values. One can notice here that the proposed structure is not very simple to be studied because its spectral responses exhibit some singularities that are very hard to handle by a theoretical or a numerical method. Nevertheless, one notes, from Fig. 5(a), that the FDTD results are consistent and present an intermediate spectral response compared with the other theoretical data.

These results demonstrate the good accuracy of our code. Nonetheless, we have made further calculations in order to show the versatility of our code even when the azimuthal angle is changed. Thus, Fig. 6 shows the reflectivity of the same 2D slab grating depicted in Fig. 5(d) both in TE and TM polarizations for $\theta=30^{\circ}$ with respect to $\phi$.

We also compare these last results with the published ones by Attiya and Kishk in Ref. [17] calculated at a fixed value 

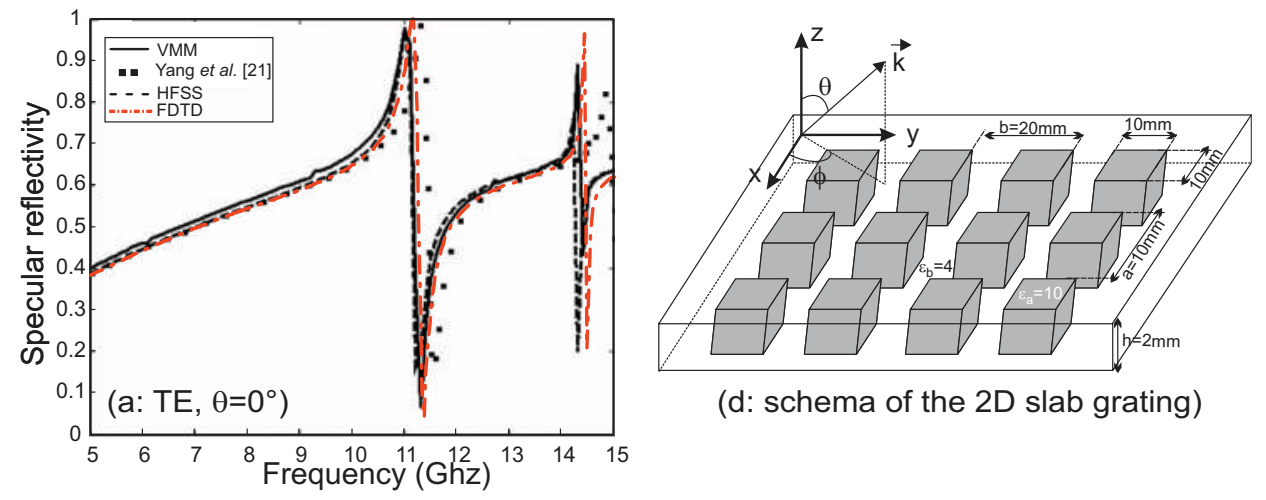

(d: schema of the 2D slab grating)
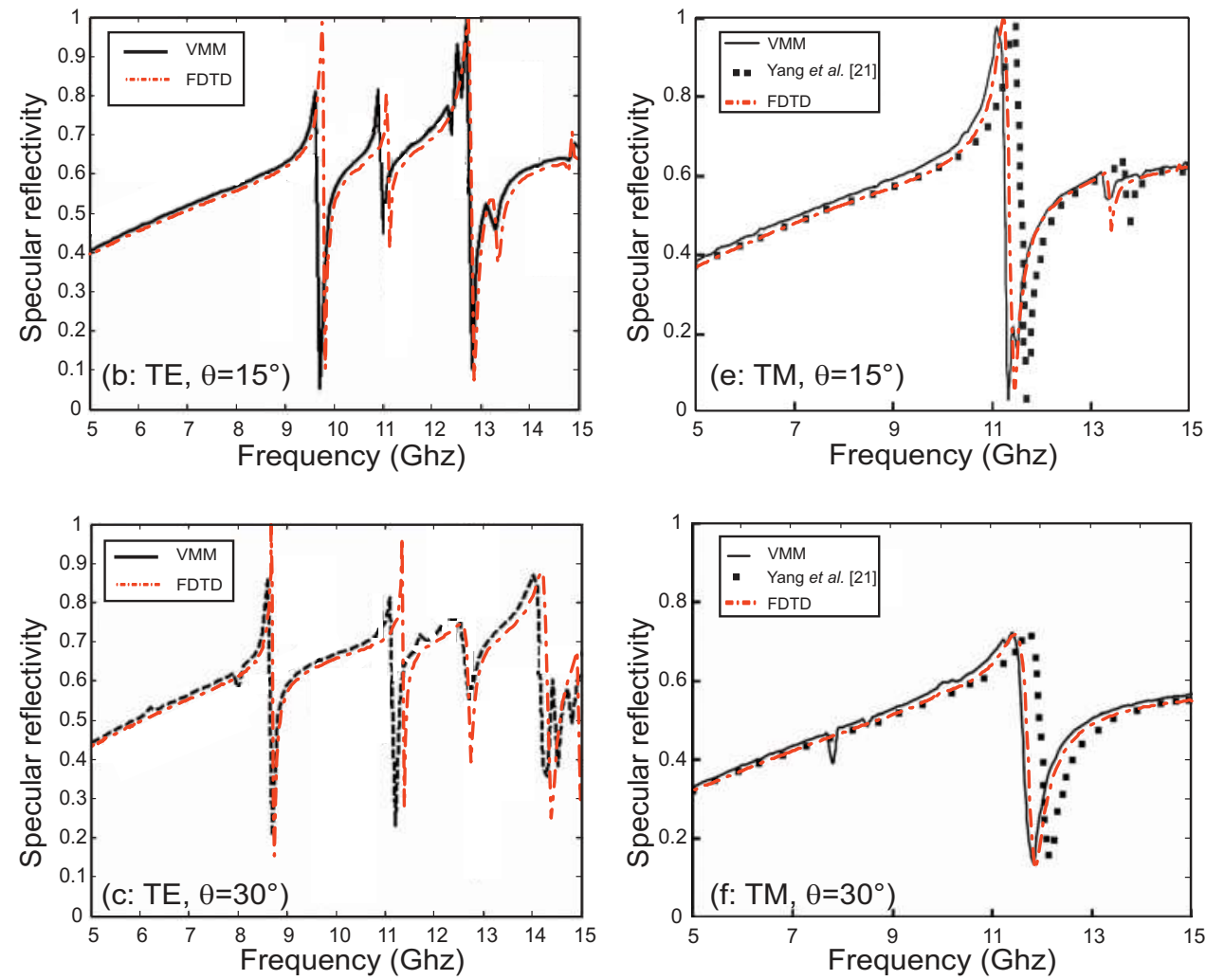

FIG. 5. (Color) FDTD results for the $2 \mathrm{D}$ slab grating depicted in (d). The first column (a),(b),(c) corresponds to the TE polarization, while the two last parts (e) and (f) show the TM case. For all the five cases, $\phi$ is fixed to $0^{\circ}$ and comparisons with already published results are shown. Note that the HFSS results are also obtained by Attiya and Kishk [17]. of the frequency, i.e., $f=10 \mathrm{Ghz}$. For this purpose, we make cross sections over the two plots of Fig. 6 and we obtain the two curves presented in Fig. 7. At first sight, one can note a large discrepancy between the two results. This difference can be related to the spatial discretization because the FDTD becomes accurate only if this spatial step tends to zero. Nevertheless, we have verified the convergence of the obtained data versus the spatial step.

However, the overall behavior of the FDTD curves in Fig. 7 is very similar to the one of the VMM method and confirms the accuracy of our code. We think that it is necessary to make other tests for the $\phi$ dependence using another rigorous method such as the FMM.

\section{APPLICATION: STUDY OF A RADOME}

In this last section, we present an original study of the enhanced transmission through an annular aperture array (AAA) made in a perfect conductor versus both angle of incidence and polarization. This study was done recently in the case of a real metal [6] (silver, for instance) and similar performances for perfectly conducting structure must be demonstrated in order to confirm the same potentialities of the AAA in the terahertz or in the millimeter-wave domains.

In fact this particular structure, which has been proposed by Baida et Van Labeke in 2002, presents a very high transmission that can be qualified by superenhanced transmission because it can reach $95 \%$. This was theoretically demonstrated following the interesting findings of Ebbesen on the transmission by surface plasmon resonance through subwavelength hole arrays engraved into a metallic layer. For the AAA structure, the enhanced transmission is due to the resonance of a guided mode through each annular aperture. The cutoff wavelength of the guided mode inside the aperture depends on the geometrical parameters of the coaxial cavities (inner and outer radii, metal thickness, and with a smaller impact the period of the array). In addition, the nature of the metal plays a key role via its dispersion. For example, the cutoff wavelength of the guided mode can be 

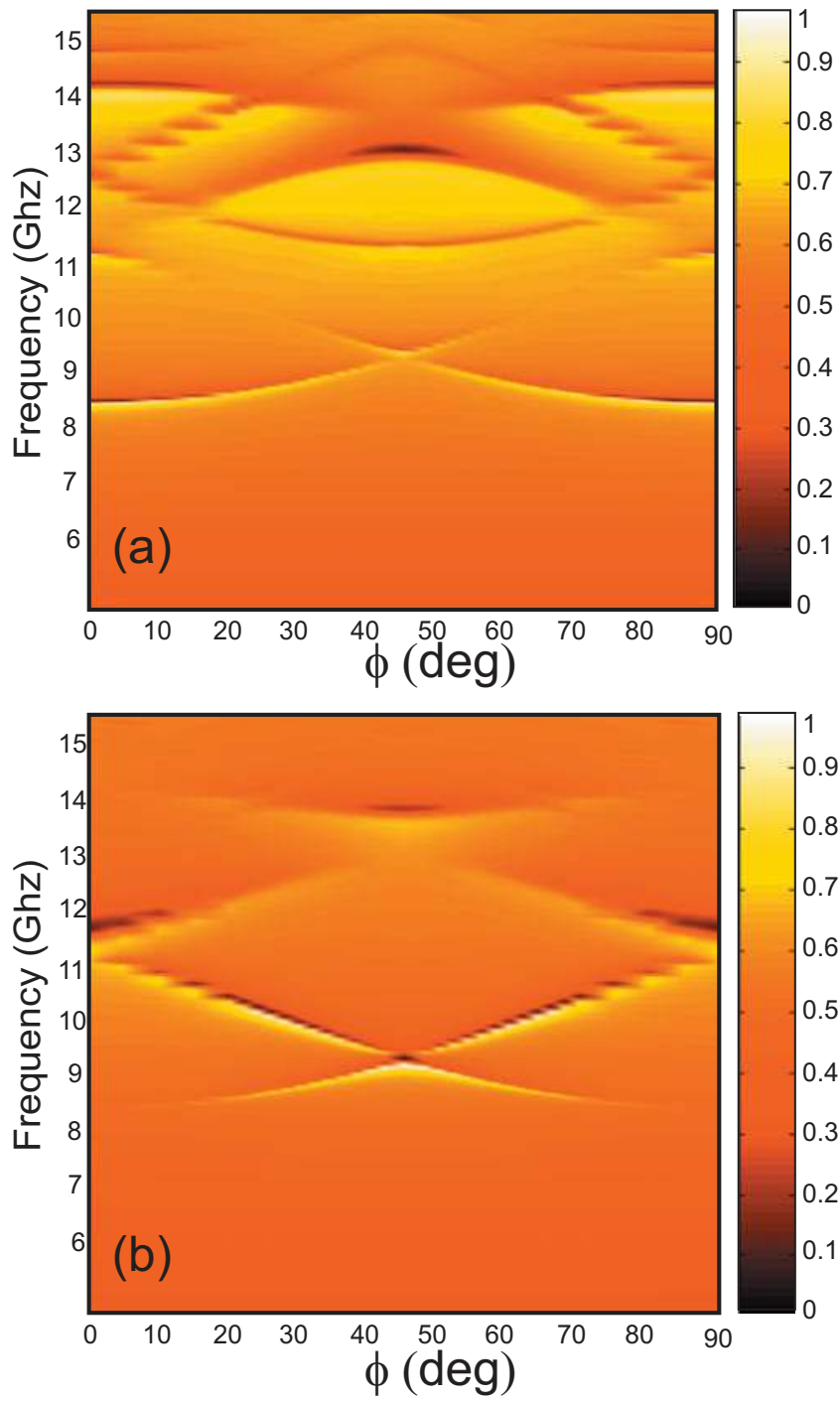

FIG. 6. (Color) Calculated specular reflectivity by the FDTD code of the 2D slab grating in TE (a) and TM (b) polarizations versus $\phi$. The calculations were done between $\phi=0^{\circ}$ and $\phi=60^{\circ}$ in order to verify the symmetry of the obtained results, and then they were extrapolated to $\phi=90^{\circ}$. For the two cases, $\theta$ is set to $30^{\circ}$.

shifted 30\% toward the red region when perfect conductor is replaced by silver.

Accordingly, AAAs present a lot of potential applications in the electromagnetic range of the spectrum where noble

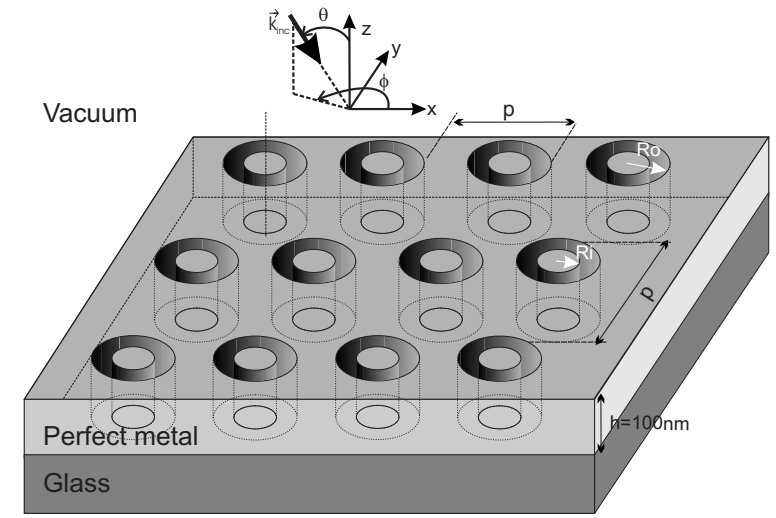

FIG. 8. Schematic of the AAA studied structure. The metallic film is deposited on a glass substrate and it is illuminated from the vacuum side by a linearly polarized plane wave.

metals are perfectly conducting (far infrared, terahertz, and millimeter wave). Particularly, the radar radome is one of the most interesting applications of such a structure. For this purpose, AAA must present the same transmission response whatever are the polarization and the angle of incidence both for the incoming and the outgoing beams. This property, that was already demonstrated for AAA structure made in silver [6], will be extended here to the perfect conductor metals that correspond to the all noble metals behavior in the military frequency range (10 Ghz-14 Ghz).

For this purpose let us consider the structure depicted in Fig. 8. It consists of a 2D array of annular apertures perforated into a $h=3.6 \mathrm{~mm}$ thick perfect metal. The values of the inner and the outer radii were chosen in order to have a transmission peak in the desired range. Thus, we put $R_{i}$ $=2.7 \mathrm{~mm}, R_{o}=3.6 \mathrm{~mm}$, and we fix the period of the array to $p=12.6 \mathrm{~mm}$ in order to cancel the Rayleigh anomaly at normal incidence. In fact, this last appears when the first diffracted order lies the grating surface, i.e., when the tangential component of the wave vector associated with this first diffracted order is equal to $\omega / v$ with $v$ being the light velocity in the incident or in the transmission media.

In order to describe the fine details of the structure, a nonuniform meshing is also used in the algorithm. It allows us to faithfully characterize the geometrical features of the apertures and of the metallic film.

Figures 9(a) and 9(b) present the transmission spectra for
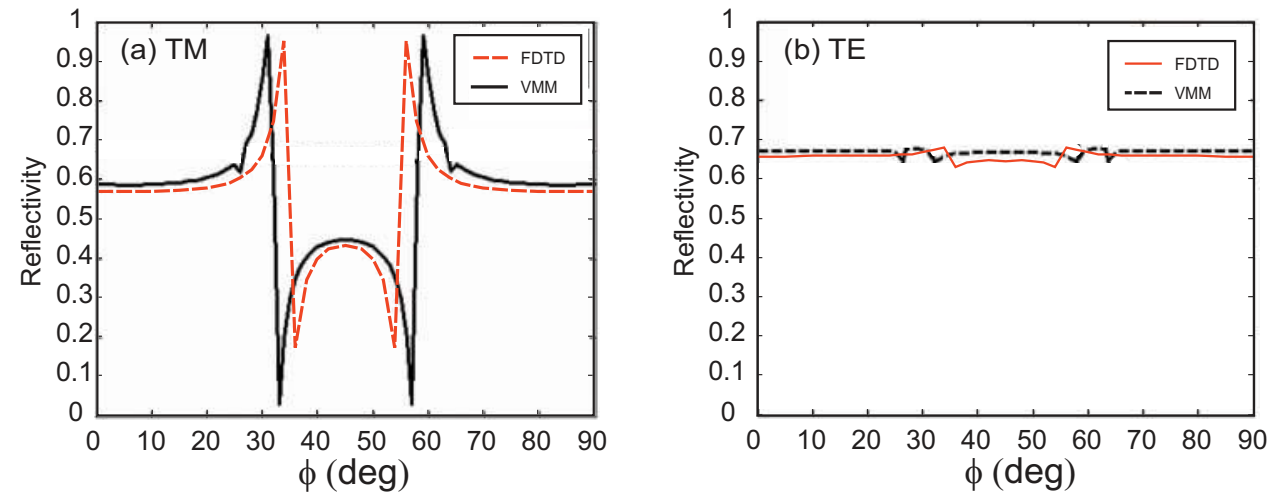

FIG. 7. (Color) Horizontal cross sections made at $f=10 \mathrm{Ghz}$ over the two plots of Fig. 6. Comparison with published results by Attiya and Kishk [17]. 

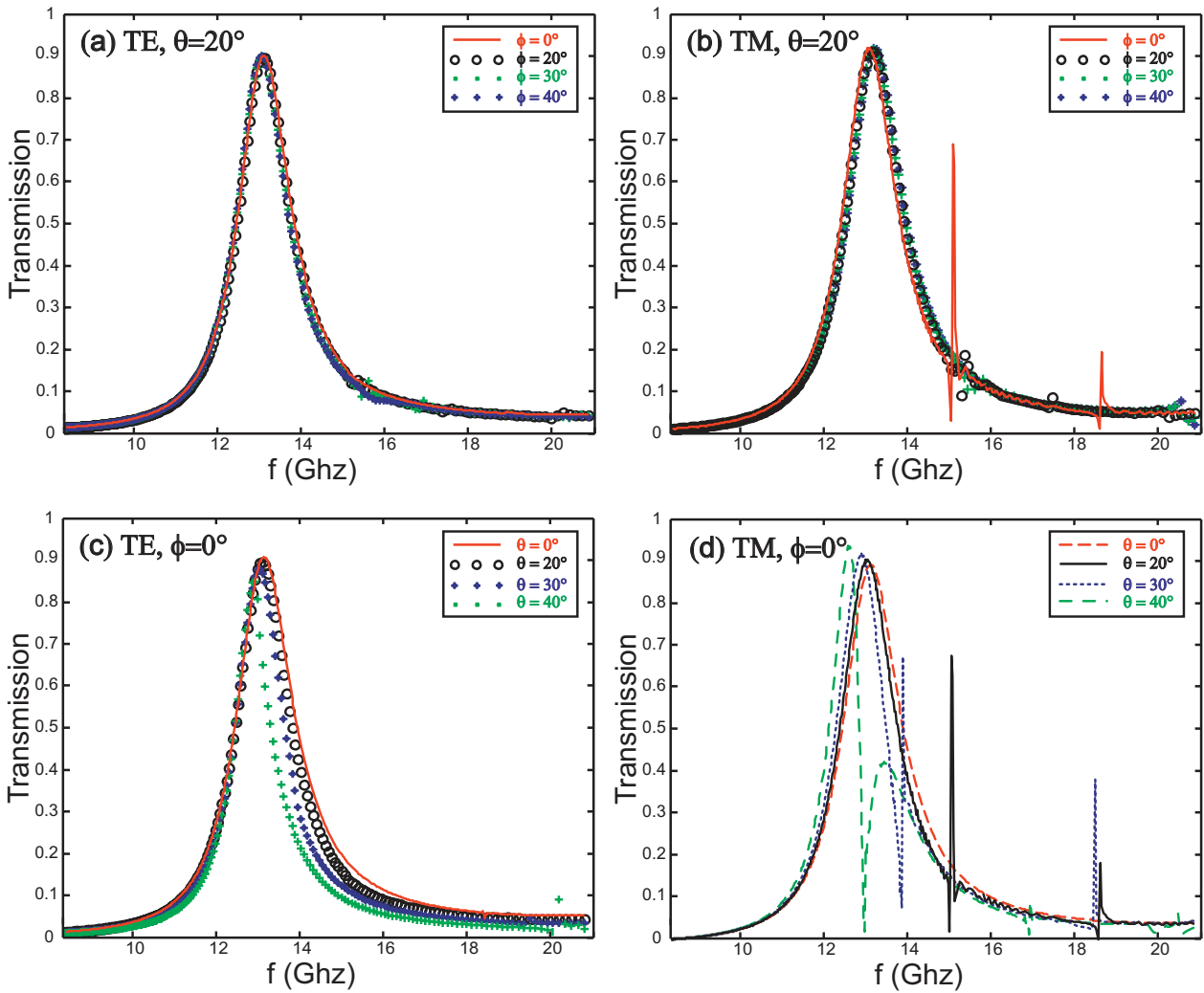

FIG. 9. (Color) Transmission spectra of the AAA structure depicted in Fig. 8 with $R_{O}=3.6 \mathrm{~mm}$, $R_{i}=2.7 \mathrm{~mm}, p=12.6 \mathrm{~mm}$, and $h$ $=3.6 \mathrm{~mm}$. (a), (b) When the azimuthal angle varies. (c),(d) When the incidence angle changes. (a),(c) correspond to TE polarization, while (b),(d) are linked to TM polarization. four different values of the azimuthal angle $(\phi)$, respectively, in the cases of TE and TM polarized incident plane waves. For the two cases, the angle of incidence is fixed at $\theta=20^{\circ}$. One can notice that, except the occurrence of Rayleigh anomalies, the transmission response of the structure remains quite identical when both polarization and azimuthal angle vary. In addition, and because of the Cartesian spatial meshing even if it is nonuniform, the contours of the annular apertures still present staircase artifacts that lead to small alterations on the transmission response when the illumination parameters change.

Finally, the robustness of the AAA structure in the transmission mode is verified also when the angle of incidence $\theta$ is varied. Figures 9(c) and 9(d) present the transmission spectra for the two polarizations (TE and TM, respectively) when the angle of incidence is varied for zero up to $40^{\circ}$. One can note that in the TM case, the influence of the Rayleigh anomalies appears as discontinuities and alters the transmission spectra. To bypass this problem, the geometrical parameters of the AAA structure should be defined in order to drive away these anomalies from the transmission peak [6].

\section{CONCLUSION}

The 3D-FDTD algorithm is adapted for oblique incidence in the case of periodic structures. Both PML ABCs and spatial nonuniform meshing are incorporated. According to us, this is an essential and unavoidable numerical tool for the study of light interaction with subwavelength structures. Tests were presented in order to demonstrate the accuracy of this algorithm. An original application is also performed with this code and shows a very interesting transmission property of annular aperture arrays. We are working to extend this algorithm to incorporate a dispersion model (Drude or Drude-Lorentz model) for the consideration of dispersive material such as noble metals in the visible range. This last point is of major interest because it allows the study of light interaction with metallodielectric structures that are extensively used in nanooptics.

\section{ACKNOWLEDGMENTS}

This work is partly supported by the "Réseau de Nanotechnologie C'NANO Grand Est." The grant of A.B. is funded by international collaboration between France and Algeria.
[1] E. Yablonovitch, Phys. Rev. Lett. 58, 2059 (1987).

[2] J. Sajeev, Phys. Rev. Lett. 58, 2486 (1987).

[3] T. W. Ebbesen, H. J. Lezec, H. F. Ghaemi, T. Thio, and P. A. Wolff, Nature (London) 391, 667 (1998).
[4] F. I. Baida and D. Van Labeke, Opt. Commun. 209, 17 (2002).

[5] T. K. Wu, Frequency Selective Surface and Grid Array (Wiley, New York, 1995).

[6] D. Van Labeke, D. Grard, B. Guizal, F. I. Baida, and L. Li, 
Opt. Express 14, 11945 (2006).

[7] K. Yee, IEEE Trans. Antennas Propag. 14, 302 (1966).

[8] A. Taflove and S. C. Hagness, Computational Electrodynamics, the Finite-Difference Time-Domain Method, 2nd ed. (Artech House, Norwood, MA, 2005).

[9] W.-J. Tsay and D. M. Pozar, IEEE Microw. Guid. Wave Lett. 3, 250 (1993).

[10] A. Alexanian, N. J. Kolias, R. C. Compton, and R. A. York, Microwave Opt. Technol. Lett. 6, 138 (1996).

[11] F. I. Baida and D. Van Labeke, Phys. Rev. B 67, 155314 (2003).

[12] M. E. Veysoglu, R. T. Shin, and J. A. Kong, J. Electromagn. Waves Appl. 7, 1595 (1993).

[13] J. A. Roden, S. D. Gedney, M. P. Kesler, J. G. Maloney, and P. H. Harms, IEEE Trans. Microwave Theory Tech. 46, 420
(1998).

[14] G. Zheng, A. A. Kishk, A. W. Glisson, and A. B. Yakovlev, PIER 59, 85 (2006).

[15] C. Oh and M. J. Escuti, Opt. Express 14, 11870 (2006).

[16] R. Petit, Electromagnetic Theory of Gratings, Topics in Current Physics, Vol. 22 (Springer-Verlag, Berlin, 1980).

[17] A. M. Attiya and A. A. Kishk, PIER 60, 221 (2006).

[18] A. Aminian and Y. Rahmat-Samii, IEEE Trans. Antennas Propag. 54, 1818 (2006).

[19] B.-I. Wu, E. Yang, J. A. Kong, J. A. Oswald, K. A. McIntosh, L. Mahoney, and S. Verghese, Microwave Opt. Technol. Lett. 27, 81 (2000).

[20] J. P. Berenger, J. Comput. Phys. 114, 185 (1994).

[21] H.-Y. D. Yang, R. Diaz, and N. G. Alexopoulos, J. Opt. Soc. Am. B 14, 2513 (1997). 